\title{
Pulmonary Embolism in Autosomal Dominant Polycystic Kidney Patient Induced by Inferior Vena Cava Mechanical Compression
}

\author{
Maamoun Basheer ${ }^{1}$, Elias Saad ${ }^{1,2}$, Nimer Assy ${ }^{1,2}$ \\ ${ }^{1}$ Internal Medicine Department, Galilee Medical Center, Nahariya, Israel \\ ${ }^{2}$ Faculty of Medicine in the Galilee, Bar-Ilan University, Safad, Israel
}

Received: 09/07/2021

Accepted: $23 / 07 / 2021$

Published: $25 / 08 / 2021$

How to cite this article: Basheer M, Saad E, Assy N. Pulmonary embolism in autosomal dominant polycystic kidney patient induced by inferior vena cava mechanical compression. EJCRIM 2021;8: doi:10.12890/2021_002767.

Conflicts of Interests: The authors declare there are no competing interests.

This article is licensed under a Commons Attribution Non-Commercial 4.0 License

\section{ABSTRACT}

Introduction: Autosomal dominant polycystic kidney disease is a common syndrome. Renal and hepatic cysts can cause discomfort, bleeding, rupture, infection, hypertension and a mass effect with compression of adjacent organs.

Case presentation: A 48-year-old man with polycystic kidney disease and hypertension presented to the emergency department for bilateral flank pain. An abdominal computed tomography scan with contrast showed a $7 \mathrm{~cm}$ heterogeneous process posteriorly and laterally to the right kidney. It appeared to be a renal cyst associated with bleeding and bilateral pulmonary artery filling defects, apparently due to pulmonary embolism. Cavography following inferior vena cava filter insertion did not show any deep vein thrombosis.

Discussion and conclusion: The pulmonary embolism was probably caused by extrinsic inferior vena cava compression by a liver cyst. Virchow's triad of stasis, vessel damage and hypercoagulability probably resulted in a thrombus which moved on the right side to the pulmonary artery.

\section{LEARNING POINTS}

- Autosomal dominant polycystic kidney disease is a common syndrome.

- Renal and hepatic cysts can compress adjacent organs.

- The mass effect of a large cyst on the right side compressed the inferior vena cava, resulting in Virchow's triad of stasis, vessel damage and hypercoagulability, which can cause pulmonary embolism.

\section{KEYWORDS}

Autosomal dominant polycystic kidney disease, renal cyst, inferior vena cava, pulmonary embolism

\section{INTRODUCTION}

Autosomal dominant polycystic kidney disease is one of the more common of the rare monogenic kidney disorders and occurs in approximately 1 in 1000 live births ${ }^{[1-3]}$. These patients have multiple liver cysts, pancreatic and splenic cysts, intracranial berry aneurysms, cardiac problems and hypertension. Complications of renal and hepatic cysts include pain, bleeding, rupture, infection, hypertension and a mass effect with compression of adjacent organs ${ }^{[4]}$. As the number of cysts increases in both kidneys, renal function progressively deteriorates and approximately half of patients reach end-stage renal disease by the age of 60 years ${ }^{[4,5]}$.

\section{CASE DESCRIPTION}

In this case report we describe a 48-year-old man with autosomal dominant polycystic kidney disease, chronic kidney disease stage 2 and 
hypertension, who presented to the emergency department for bilateral flank pain after outpatient treatment for a urinary tract infection 2 weeks previously. The patient reported progressive lower back pain. He did not report any dyspnoea, fever, cough or gastrointestinal symptoms.

In the emergency department, the patient presented with haemodynamic and respiratory stability, without fever. A physical examination revealed bilateral lower back tenderness. An abdominal computed tomography scan with contrast showed a $7 \mathrm{~cm}$ heterogeneous process located posteriorly and laterally to the right kidney, which merged with the kidney at the lower back, forming a renal cyst which subsequently bled. Multiple hypodense lesions scattered throughout the liver were also evident. Another finding was bilateral pulmonary artery filling defects, suggesting pulmonary disease. A computed tomography angiography scan confirmed these findings (Fig. 1). Due to concern about the bleeding cyst, an inferior vena cava filter was inserted. No deep vein thrombosis was evident as documented by cavography.

The right kidney and liver cysts formed two pressure points that compressed the inferior vena cava, causing mechanical stress further leading to thrombosis. An embolus moved to the pulmonary arteries.

Evaluation for underlying inherited and acquired hypercoagulable states revealed normal results. Laboratory biochemistry tests showed creatinine levels at $1.6 \mathrm{mg} / \mathrm{dl}$ (baseline $1.5-1.6 \mathrm{mg} / \mathrm{dl}$ ). A complete blood cell count was within the normal range. Magnetic resonance imaging also showed no cerebral aneurysms.
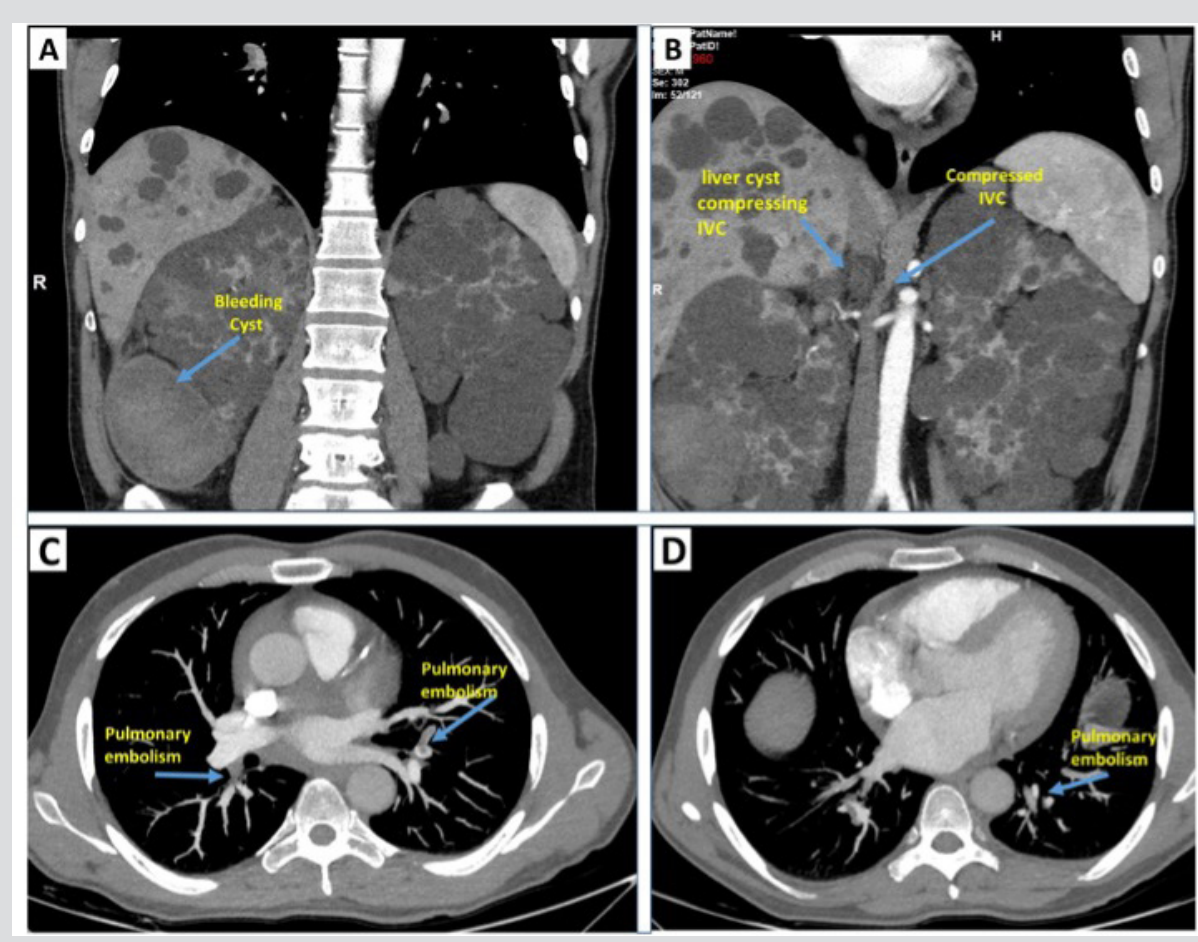

Figure 1. Computed tomography: (A) a bleeding cyst; (B) right liver cyst compressing the inferior vena cava (IVC); (C, D) pulmonary artery filling defects

A dimercaptosuccinic acid mapping kidney test showed non-homogeneous absorption and many filling defects in both kidneys. The glomerular filtration rate contribution was $38 \%$ and $62 \%$ in the right and the left kidney, respectively. A general surgeon and a urologist both examined the patient and recommended conservative treatment.

\section{DISCUSSION}

We present a case of autosomal dominant polycystic kidney disease in a patient who presented with pulmonary disease without evidence of deep vein thrombosis in the lower extremities. The patient was discharged on anticoagulation therapy. Angiography following filter insertion, showed two narrowed areas near the right kidney and the liver. The mass effect of a large cyst on the right side induced compression of the inferior vena cava. Virchow's triad of stasis, vessel damage and hypercoagulability may have resulted in thrombosis in the inferior vena cava and an embolus which moved on the right side to the pulmonary artery.

There have been few cases reports on this topic. A 37-year-old man with known polycystic kidney disease was found unresponsive at home. The autopsy revealed an extensive inferior vena cava thrombus with extrinsic compression caused by a right renal cyst. There were no signs 
of iliofemoral vessel disease or deep vein thrombosis. The postulated mechanism of death was inferior vena cava compression due to a large right renal cyst leading to venous stasis and thrombosis. This resulted in pulmonary embolism and cardiovascular collapse ${ }^{[6]}$.

Morimoto and colleagues also showed that tolvaptan, which prevents renal cyst development, could cause acute pulmonary thromboembolism during treatment for autosomal dominant polycystic kidney disease ${ }^{[7]}$. A few other cases discussed the same mechanism of pulmonary embolism due to a large cyst that compressed the inferior vena cava ${ }^{[8-13]}$.

The suggested mechanism of a mass effect of a large liver cyst compressing the inferior vena cava causing venous stasis and local endothelial injury is shown in Fig. 1. Damage to the endothelium can contribute to Virchow's triad. When the endothelium is damaged, the secretion of pro-coagulants is increased and the secretion of vasodilators is decreased, leading to thrombosis in the inferior vena cava complicated with pulmonary embolism.

The risk of death in untreated deep vein thrombosis or pulmonary embolism exceeds $30 \%$. Furthermore, bleeding from cysts in polycystic kidney patients is common. Up to $70 \%$ of patients develop cyst haemorrhage and haematuria ${ }^{[14,15]}$. The risk of haemorrhage rises with increasing kidney volume, especially if the kidneys are over $15 \mathrm{~cm}$ in length ${ }^{[16,17]}$. We believe anti-coagulation therapy is necessary in a case of documented pulmonary embolism because failure to administer this treatment can lead to sudden death. On the other hand, bleeding from cysts most commonly presents as gross haematuria, which serves as a good predictor for diagnosing and treating the condition. Further follow-up and research on this topic is needed. Patients with this syndrome are at risk for severe complications. The question arises as to whether nephrectomy is necessary in patients with an impaired kidney but a normal glomerular filtration rate.

\section{CONCLUSION}

We describe the case report of young man who developed pulmonary embolism without evidence of deep vein thrombosis. The mechanism was probably extrinsic compression by a right liver cyst and right kidney cyst and concomitant thrombus in the main pulmonary artery.

\section{REFERENCES}

1. Davies F, Coles GA, Harper PS, Williams AJ, Evans C, Cochlin D. Polycystic kidney disease re-evaluated: a population-based study. Q J Med 1991;79:477-485

2. Gabow PA. Autosomal dominant polycystic kidney disease. N Engl J Med 1993;329:332-342.

3. Levy M, Feingold J. Estimating prevalence in single-gene kidney diseases. progressing to renal failure. Kidney Int 2000;58:925-943.

4. Torres VE, Harris PC, Pirson Y. Autosomal dominant polycystic kidney disease. Lancet 2007;369:1287-1301.

5. Higashihara E, Nutahara K, Kojima M, Tamakoshi A, Yoshiyuki O, Sakai H, et al. Prevalence and renal prognosis of diagnosed autosomal dominant polycystic kidney disease in Japan. Nephron 1998;80:421-427.

6. Tamburrini R, Ahmed Z, van der Walt J, Goldsmith D. Sudden death of a patient with polycystic kidneys due to acute inferior vena cava thrombosis. Scott Med J 2016;61(3):171173.

7. Morimoto K, Akai Y, Matsui M, Yano H, Tagawa M, Samejima KI, et al. Acute pulmonary thromboembolism occurring during treatment with tolvaptan in a patient with autosomal-dominant polycystic kidney disease. CEN Case Rep 2017;6(1):61-65.

8. Holzmann-Littig C, Lorenz G, Wen M, Heemann U, Renders L. A case of pulmonary embolism caused by compression of the vena cava by intra-abdominal masses in autosomal polycystic kidney disease. Clin Case Rep 2020;8(7):1149-1152.

9. O'Sullivan DA, Torres VE, Heit JA, Liggett S, King BF. Compression of the inferior vena cava by right renal cysts: an unusual cause of IVC and/or iliofemoral thrombosis with pulmonary embolism in autosomal dominant polycystic kidney disease. Clin Nephrol 1998;49(5):332-334.

10. Maeda T, Uchida Y, Oyamada K, Nakajima F. Thrombosis in inferior vena cava due to enlarged renal cysts in autosomal dominant polycystic kidney disease. Intern Med 2010;49:1891-1894.

11. Ko MK, Kim T, Lee WH, Park SH, Choi JH, Shin M, et al. Deep vein thrombosis due to compression of huge hepatic cyst successfully treated by inferior vena cava filter and cyst drainage. Korean J Gastroenterol 2018;72(3):146-149.

12. Peces R, Gil F, Costero O, Pobes A. Massive inferior vena cava thrombosis in a patient with autosomal dominant polycystic hepatorenal disease. Nefrologia 2002;22(1):75-78.

13. Iguchi S, Kasai A, Kishimoto H, Suzuki K, Ito S, Ogawa Y, et al. Thrombosis in inferior vena cava (IVC) due to intra-cystic hemorrhage into a hepatic local cyst with autosomal dominant polycystic kidney disease (ADPKD). Intern Med 2004;43(3):209-212.

14. Grantham JJ. The etiology, pathogenesis, and treatment of autosomal dominant polycystic kidney disease: recent advances. Am J Kidney Dis 1996;28(6):788-803.

15. Levine E, Grantham JJ. Perinephric hemorrhage in autosomal dominant polycystic kidney disease: CT and MR findings. J Comput Assist Tomogr 1987;11(1):108-111.

16. Gabow PA. Autosomal dominant polycystic kidney disease. N Engl J Med 1993;329(5):332-342.

17. Gabow PA, Duley I, Johnson AM. Clinical profiles of gross hematuria in autosomal dominant polycystic kidney disease. Am J Kidney Dis 1992;20(2):140-143. 
European Journal

of Case Reports in

Internal Medicine 